\title{
Insulin-Containing Wound Dressing Promotes Diabetic Wound Healing Through Stabilizing HIF-1 $\alpha$
}

\author{
Peilang Yang ${ }^{1+}$, Di Wang ${ }^{2+}$, Yan Shi' ${ }^{1+}$, Mingzhong $\mathrm{Li}^{3}$, Min Gao', Tianyi Yu', Dan Liu', \\ Jie Zhang ${ }^{1}$, Jizhuang Wang ${ }^{1}$, Xiong Zhang ${ }^{1 *}$ and Yan Liu ${ }^{1 *}$ \\ 'Department of Burn and Plastic Surgery Ruijin Hospital Affiliated to Shanghai Jiao Tong University School of Medicine, \\ Shanghai, China, ${ }^{2}$ Department of Anesthesiology, Shanghai Jiao Tong University Affiliated Sixth People's Hospital, Shanghai, \\ China, ${ }^{3}$ National Engineering Laboratory for Modern Silk, College of Textile and Clothing Engineering, Soochow University, \\ Suzhou, China
}

OPEN ACCESS

Edited by:

Kai Zheng,

University of Erlangen-Nuremberg,

Germany

Reviewed by:

Qingqing Yao,

Wenzhou Medical University, China

Jun Wu,

Shenzhen Second People's Hospital,

China

*Correspondence:

Xiong Zhang

xiong@medmail.com.cn

Yan Liu

riliuyan@126.com

${ }^{\dagger}$ These authors have contributed equally to this work

Specialty section:

This article was submitted to Tissue Engineering and Regenerative

Medicine,

a section of the journal

Frontiers in Bioengineering and

Biotechnology

Received: 08 August 2020 Accepted: 23 November 2020

Published: 18 December 2020

Citation:

Yang P, Wang D, Shi Y, Li M, Gao M, Yu T, Liu D, Zhang J, Wang J,

Zhang $X$ and Liu Y (2020) Insulin-Containing Wound Dressing Promotes Diabetic Wound Healing

Through Stabilizing HIF-1 $\alpha$.

Front. Bioeng. Biotechnol. 8:592833.

doi: 10.3389/fbioe.2020.592833
$\mathrm{HIF-1 \alpha}$ is seen as a major regulator during wound healing and controls many wound healing processes, such as angiogenesis, extracellular deposition, and reepithelialization. A diabetic state plays a vicious effect on wound healing, and the destabilization of HIF-1 $\alpha$ is a non-negligible factor. Insulin-loaded silk fibroin microparticles were prepared to release insulin by covering the wounds, and this material was proven to promote wound healing in both in vitro and in vivo studies. In this work, we found that this insulin-containing wound dressing could accelerate diabetic wound healing by promoting reepithelialization, angiogenesis, and extracellular matrix, especially collagen deposition. Meanwhile, HIF-1 $\alpha$ was stable and accumulated in insulin-containing dressing to group wound cells, which was significantly unstable in the control group. In further studies, we showed that methylglyoxal (MGO), the main form of advanced glycation end products (AGEs), accumulated significantly and caused the destabilization of HIF-1 $\alpha$ in the diabetic state. Insulin could alleviate the MGO-induced $\mathrm{HIF}-1 \alpha$ unstable state and promote HIF- $1 \alpha$ target gene expression and its downstream biological effect such as angiogenesis and wound extracellular matrix deposition.

Keywords: diabetes, wound healing, insulin, HIF-1 $\alpha$, angiogenesis

\section{INTRODUCTION}

Diabetic non-healing wound is one of the most common late complication of diabetic patients. The morbidity rate of diabetic non-healing wound ranges from 5 to $20 \%$ among all diabetic patients. Diabetic wound puts a huge pressure on the social health economic system for its retarded healing characteristic (Falanga, 2005; Davis et al., 2018). Histologically, diabetic wound shows the characteristics of impaired angiogenesis, reepithelialization, and extracellular matrix deposition (Demidova-Rice et al., 2012). All these characteristics are related to an unstable HIF- $1 \alpha$ in the diabetic environment. HIF- $1 \alpha$ is considered as the central regulator of wound healing, and it dominates wound healing processes, such as inflammation reaction, angiogenesis, reepithelialization, and extracellular matrix deposition (Hong et al., 2014).

In a diabetic wound environment, the stabilization of HIF- $1 \alpha$ would be severely impaired in the hypoxia state, and the instability of HIF-1 $\alpha$ is regarded as the major factor of diabetic nonhealing wound. In the diabetic state, the instability of HIF-1 $\alpha$ is often caused by the accumulation 
of advanced glycation end products (AGEs). Recently, methylglyoxal (MGO), the major form of AGEs, has been reported to induce HIF-1 $\alpha$ instability in many ways (Ramasamy et al., 2006; Catrina, 2014). Therefore, many researches aim at stabilizing HIF-1 $\alpha$ to improve wound healing (Mace et al., 2007; Botusan et al., 2008). Our previous research results show that insulin can act as a growth-like factor to promote wound healing by promoting angiogenesis, reepithelialization, and extracellular matrix deposition (Liu et al., 2009a,b). Based on these results, in this work, we develop a wound dressing by loading insulin into silk fibroin (SF) microparticles (Li et al., 2017). As many functions of insulin on wound healing overlapped with the functions of HIF-1 $\alpha$, we in this research intend to check the effects of insulin-containing dressing material on diabetic wound healing and whether insulin takes effect through regulating HIF-1 $\alpha$ stabilization.

\section{MATERIALS AND METHODS}

\section{Reagents}

Streptozocin (STZ), insulin for cell culture, and MGO were from Sigma-Aldrich (St. Louis, MO, United States). ProLong gold antifade mounting reagent with DAPI was purchased from Thermo Fisher Scientific (Carlsbad, CA, United States). RPMI 1640, DMEM, and phosphate buffer solution (PBS) were obtained from GIBCO (Carlsbad, CA, United States). Chemiluminescence reagent was from Millipore (Billerica, MA, United States). Insulin was from Roche chemical (Houston, TX, United States). OxiSelect MGO Competitive ELISA Kit was purchased from Cell Biolabs (San Diego, CA, United States). Matrigel was from BD Biosciences (Waltham, MA, United States).

The following antibodies were obtained from various suppliers: PECAM-1, collagen I, and collagen III were from Abcam (Cambridge Science Park, Cambridge); CBP, GLUT-1, VEGF-A, HIF-1 $\alpha$, and HRP-labeled secondary antibody were from Cell Signaling Technology (Danvers, MA, United States); and donkey anti-rabbit Alexa Fluor 488 was purchased from Thermo Fisher Scientific (Carlsbad, CA, United States).

\section{Animals}

Forty male Sprague-Dawley rats, 6 weeks old and weighing 120$140 \mathrm{~g}$, were purchased from Shanghai Laboratory Animal Center in the Chinese Academy of Sciences and housed at the Animal Science Center of Shanghai Jiao Tong University, School of Medicine (SJTUSM). The animals were maintained under a 12h light/dark cycle at $22^{\circ} \mathrm{C}$. Thirty rats were used to develop diabetes by a reported method. In brief, the rats were fasted for $16 \mathrm{~h}$ followed by a single dose of STZ $(60 \mathrm{mg} / \mathrm{kg}$ BW dissolved in $0.1 \mathrm{mM}$ citrate buffer) intraperitoneally (Junod et al., 1969). Rats were left to develop diabetes for 8 weeks. Random plasma glucose was measured, and a glucose level $>300 \mathrm{mg} / \mathrm{L}$ was considered diabetic. The rest of the rats were set as normal control (Norm). The animal procedures were performed in accordance with the rules of the Animal Care Committee of SJTUSM, and all experiment protocols were approved by the SJTUSM Institutional Animal Care and Use Committee.

\section{Preparation of SF Microparticles}

The preparation of SF microparticles with or without insulin was described in a previous study as follows (Li et al., 2017).

(1) Preparation of SF solution: Briefly, Bombyx mori silk fibers (Huzhou, China) were degummed three times in $0.05 \% \mathrm{Na}_{2} \mathrm{CO}_{3}$ solution at $98-100^{\circ} \mathrm{C}$ for $30 \mathrm{~min}$ and dried at $60^{\circ} \mathrm{C}$ after thorough rinsing. The fibroin extract was dissolved in a ternary solvent of $\mathrm{CaCl}_{2}: \mathrm{CH}_{3} \mathrm{CH}_{2} \mathrm{OH}: \mathrm{H}_{2} \mathrm{O}\left(1: 2: 8\right.$ molar ratio) at $72 \pm 2{ }^{\circ} \mathrm{C}$ for $1 \mathrm{~h}$. The solution was then dialyzed in cellulose tubes (MWCO 9-14 kDa) in deionized water for 4 days.

(2) Preparation of SF microparticles: $100 \mathrm{mg}$ insulin $(27.5$ IU/mg; from porcine, Wanbang Biochemical Pharmaceutical Co., Ltd., Xuzhou, China) was dissolved in $10 \mathrm{ml}$ of $0.01 \mathrm{~N} \mathrm{HCl}$; then the $\mathrm{pH}$ of the solution was adjusted to $7.0 \pm 0.1$ using $0.1 \mathrm{~N} \mathrm{NaOH}$. The SF solution was diluted to $2.0 \mathrm{wt} \%$ and mixed with glycerol at 30 wt.\% of SF weight. The resulting insulin solution and SF solution were used as core and shell for coaxial electrostatic differentiation, respectively.

(3) Preparation of microparticle-loaded SF sponge dressing: The SF solution was diluted to $2.0 \mathrm{wt} . \%$, and then 2-morpholinoethanesulfonic acid/N-hydroxysuccinimide/1ethyl-3-(3-dimethylaminopropyl)carbodiimide hydrochloride (MES/NHS/EDC) was added to the SF solution at 20/10/20 wt.\% of SF weight, respectively. Nine hundred microliters of the above mixed solution was added into a round aluminum box $(D=40 \mathrm{~mm}, H=15 \mathrm{~mm}, V=10 \mathrm{ml})$ and frozen at $-80^{\circ} \mathrm{C}$ for $30 \mathrm{~min}$. Then 10-mg SF microparticles were evenly laid on a frozen SF solution layer and frozen at $-80^{\circ} \mathrm{C}$ for $15 \mathrm{~min}$. On top of it, $700 \mu \mathrm{l}$ of the above mixed solution was added to cover the microparticles and frozen at $-80^{\circ} \mathrm{C}$ for $15 \mathrm{~min}$, followed by 10-mg SF microparticles and $900 \mu \mathrm{l}$ SF solution at a similar process. Then the SF mixture composite with two layers of microparticles was frozen at $-80^{\circ} \mathrm{C}$ for $4 \mathrm{~h}$ and further freeze-dried to obtain microparticle-loaded SF sponge dressing. The dressings were sterilized with $\gamma$-ray irradiation and stored at $4^{\circ} \mathrm{C}$.

\section{Scanning Electron Microscopy}

The morphology of samples was observed by a scanning electron microscope (SEM, S-4800, Hitachi, Japan) described as before ( $\mathrm{Li}$ et al., 2017). The size of microparticles was analyzed on the basis of SEM images with the Nano Measurer analysis software (Department of Chemistry, Fudan University, China. 2008 Jie Xu).

\section{In vitro Insulin Release}

The in vitro insulin release was determined as described before (Zhao et al., 2012; Li et al., 2017). Insulin was labeled with fluorescein isothiocyanate (FITC) (Sigma-Aldrich). Three hundred microliters of FITC solution $(10 \mathrm{mg} / \mathrm{ml}$ in dimethyl sulfoxide) was added to $10 \mathrm{ml}$ of insulin solution (15 $\mathrm{mg} / \mathrm{ml}$ in bicarbonate buffer, $\mathrm{pH}=8.5,0.1 \mathrm{M})$ and stirred at room temperature for $60 \mathrm{~min}$. Next, $200 \mu \mathrm{l}$ of $1 \mathrm{M}$ hydroxylammonium chloride solution was added and stirred for $10 \mathrm{~min}$ at room temperature. The insulin was then purified using a $10 \mathrm{~mm} \times 300 \mathrm{~mm}$ column with Sephadex G-50 
equilibrated in $0.1 \mathrm{M}$ sodium bicarbonate buffer $(\mathrm{pH}=8.5)$ to remove any unreacted FITC. The fluorescent images were captured using an inverted fluorescence microscope (Olympus IX71, Japan). Physically absorbed microparticles were used as a control group. Ten milligrams of pure SF microparticles was directly immersed into $2 \mathrm{ml}$ of FITC-insulin solution to adsorb insulin by permeation and physical absorption, which were named insulin-adsorbed microparticles. The remaining amount of insulin in solution was quantified to calculate the adsorption amount using a fluorescence spectrophotometer (FM4P TCSPC, Horiba Jobin Yvon), and the loading ratio in the insulinadsorbed microparticles was about 5.5\%. The insulin release profiles were determined by immersing $10 \mathrm{mg}$ of microparticles and a sponge dressing containing $10 \mathrm{mg}$ of microparticles in $10 \mathrm{ml}$ of PBS (10 mM, pH 7.4). The samples were incubated in a water bath at $37^{\circ} \mathrm{C}$ with a 100 -rpm shaking. One milliliter of medium was collected and replaced with an equal volume of fresh PBS after centrifugation at predetermined time points. The amount of released insulin was quantified using a fluorescence spectrophotometer.

\section{Wounding Procedure}

One day before wounding, rats were anesthetized with a single intraperitoneal injection of thiopental sodium $(40 \mathrm{mg} / \mathrm{kg} \mathrm{BW})$; the hairs on the back were shaved and thoroughly removed using the Nair hair remover lotion. On the day of wounding, the rats were anesthetized with thiopental sodium and a dose of tramadol. The animals' backs were incised to produce a $1.5 \mathrm{~cm} \times 1.5 \mathrm{~cm}$ wound.

In the first part, the rats were separated into two groups: the normal non-diabetic group (Norm) and the diabetic group (DM). In the second part, the rats were also separated into two groups: the group in which the diabetic wound was dressed with SF microparticles without insulin (Ctrl) and the group in which the diabetic wound was dressed with SF microparticles containing insulin (INS).

\section{Measurement of Wound Closure}

Rats were anesthetized with thiopental sodium at the 5th and 11th days after wounding. The wounds were photographed and drawn on transparent tracing papers, and the papers were scanned. The wound sizes were analyzed using ImageJ software. The unhealed rate was calculated by comparing the unhealed wound area to the original wound area.

\section{Histological Observation}

The wounds, including $5 \mathrm{~mm}$ of adjacent normal skin, along with the subcutaneous fat tissue were harvested at the 5th and 11th days after wounding. The tissue was fixed in $4 \%$ paraformaldehyde and embedded in paraffin. Sections with 6-7 $\mu \mathrm{m}$ thickness were stained with hematoxylin and eosin (H\&E) for histological and morphometric observation and evaluation. Masson trichrome staining was used for collagen deposition evaluation.

\section{Immunohistochemistry}

The wounds were harvested and fixed as mentioned above, and sections were then deparaffinized, rehydrated, and washed in distilled water. The sections were placed in $95 \sim 98^{\circ} \mathrm{C}$ antigen retrieval citrate buffer in a container for 10-15 min. Endogenous peroxidase activity was blocked by placing the sections in $3 \%$ hydrogen peroxide in methanol for $10 \mathrm{~min}$. Non-specific staining was blocked with normal goat serum, and the sections were incubated with anti-rat PECAM-1, VEGF-A, or HIF$1 \alpha$ overnight at $4^{\circ} \mathrm{C}$. After washing, HRP-labeled secondary antibody was applied for $1 \mathrm{~h}$ at room temperature and then with diaminobenzidine and counterstained with hematoxylin.

\section{Immunofluorescence}

Cells were washed with PBS three times and fixed with $4 \%$ paraformaldehyde in PBS at a $\mathrm{pH}$ of 7.4 for $5 \mathrm{~min}$. The cells were incubated for 10 min with PBS containing $0.25 \%$ Triton $\mathrm{X}-100$. Non-specific staining was blocked with normal goat serum, and the sections were incubated with anti-human HIF$1 \alpha$ overnight at $4^{\circ} \mathrm{C}$. After washing, Alexa Fluor 488-labeled secondary antibody was applied for $1 \mathrm{~h}$ at room temperature. Coverslips were mounted with a drop of ProLong gold antifade mounting reagent with DAPI and sealed with nail polish to prevent drying and movement under the microscope.

\section{Western Blot}

The tissues were homogenized by pulverization in liquid nitrogen and transferred to tissue lysis buffer with a protease inhibitor cocktail following centrifugation at $12,000 \mathrm{rpm}$ for $15 \mathrm{~min}$. The supernatants were removed and stored at $-80^{\circ} \mathrm{C}$. An equal amount of protein per lane $(50 \mu \mathrm{g})$ was separated by $5-12 \%$ SDSPAGE and transferred to a polyvinylidene difluoride membrane. The membranes were blocked by $5 \%$ non-fat powdered milk in Tris-buffered saline with Tween-20 (TBST) and then incubated with anti-PECAM-1, collagen I, collagen III, VEGF-A, CBP, GLUT-1, and HIF- $1 \alpha$ primary antibody in 5\% non-fat milk in TBST overnight at $4^{\circ} \mathrm{C}$. The membrane was then washed extensively with TBST and then incubated with the secondary antibody for $1 \mathrm{~h}$ at room temperature. Bands were visualized with enhanced chemiluminescence. Relative quantities of protein were determined using a densitometer and presented in comparison with $\beta$-actin expression.

\section{Cell Culture}

HUVECs and fibroblasts were purchased from ATCC and cultured as recommended.

\section{HUVEC Tube Formation Assay}

The tube formation assays were operated according to the manufacturer's instruction. Briefly, the Matrigel was thawed overnight on ice at $4^{\circ} \mathrm{C}$, and the vial was swirled to ensure that the material is evenly dispersed when the Matrigel thawed. Chilled Matrigel $(10 \mathrm{mg} / \mathrm{ml})$ was added at $0.3 \mathrm{ml}$ per well with precooled tips, and the entire growth surface of 24 -well culture plates were covered completely and evenly. Plates were incubated at $37^{\circ} \mathrm{C}$ for $45 \mathrm{~min}$. The remaining liquid was carefully removed 
from the culture well without disturbing the layer of Matrigel just before use. Passage 4 HUVECs were detached, and the cells were resuspended in culture medium with 5 serum at $1 \times 10^{6}$ cells $/ \mathrm{ml}$ with or without $800 \mu \mathrm{M}$ MGO or with $800 \mu \mathrm{M}$ MGO and $10^{-6}$ $\mathrm{M}$ insulin. Into each well was added $300 \mu \mathrm{l}$ of the cell suspension, and the angiogenesis assay plate was incubated for $12 \mathrm{~h}$ at $37^{\circ} \mathrm{C}$, at $5 \% \mathrm{CO}_{2}$ atmosphere. Cells were monitored and photographed using an inverted microscope.

\section{Statistical Analysis}

Data analysis was performed using GraphPad Prism software (GraphPad Software Inc). T-tests were used to determine the significance of pairwise differences between means, unpaired $T$-tests was used for comparison between two groups, and one-way ANOVA (Dunnett's post hoc test) was used to determine significance between means of several groups. Data satisfying the assumptions of ANOVA were verified before performing the tests. A $p$-value less than 0.05 was considered significant statistically, and a $p$-value less than 0.01 was considered statistically highly significant. Data are shown as mean \pm standard deviation (SD).

\section{RESULTS}

\section{Diabetic Wounds Showed Significantly Retarded Healing Characteristics}

Compared with normal wounds, diabetic wounds showed an obviously delayed wound healing feature. As shown in Figure 1A, the reepithelialization of diabetic wounds was significantly slower than that of normal wounds. On the 11th day, when normal wounds show an almost completed reepithelialization, there still existed a large area of un-epithelialization in the diabetic wound. Meanwhile, the angiogenesis of diabetic wounds was also significantly disrupted in the diabetic state, and it was characterized as aberrant, with a leaky neovascular network and lower density compared with the normal wound on the fifth day (Figure 1B). The collagen deposition was examined by Masson trichrome staining, which showed lower collagen deposition in diabetic wounds (Figures 1C,D). The granular tissue representing the healing quality and extracellular matrix deposition also proved that the diabetic wound healing process was significantly impaired. The thickness of the granular tissue in the diabetic wound healing group was much lower than that of the normal group as shown in Figures 1E,F. As the main regulator of wound healing, $H I F-1 \alpha$ was significantly unstable in diabetic wounds, which resulted in the non-healing feature of diabetic wounds. In the wounds of our diabetic model, we confirmed that HIF-1 $\alpha$ was extremely unstable. As shown in Figures $\mathbf{1 G}, \mathbf{H}$, we found that the HIF-1 $\alpha$ expression in the diabetic wound was significantly lower than that in normal wound.

\section{Insulin-Containing Wound Dressing Promotes Diabetic Wound Healing}

Based on our previous research results, topical insulin application could accelerate wound healing, but it had some shortcomings such as the inconvenience of insulin daily application and disruption of glucose level. Based on these reasons, we composed insulin-loaded SF microparticles. The SEM characteristics of this

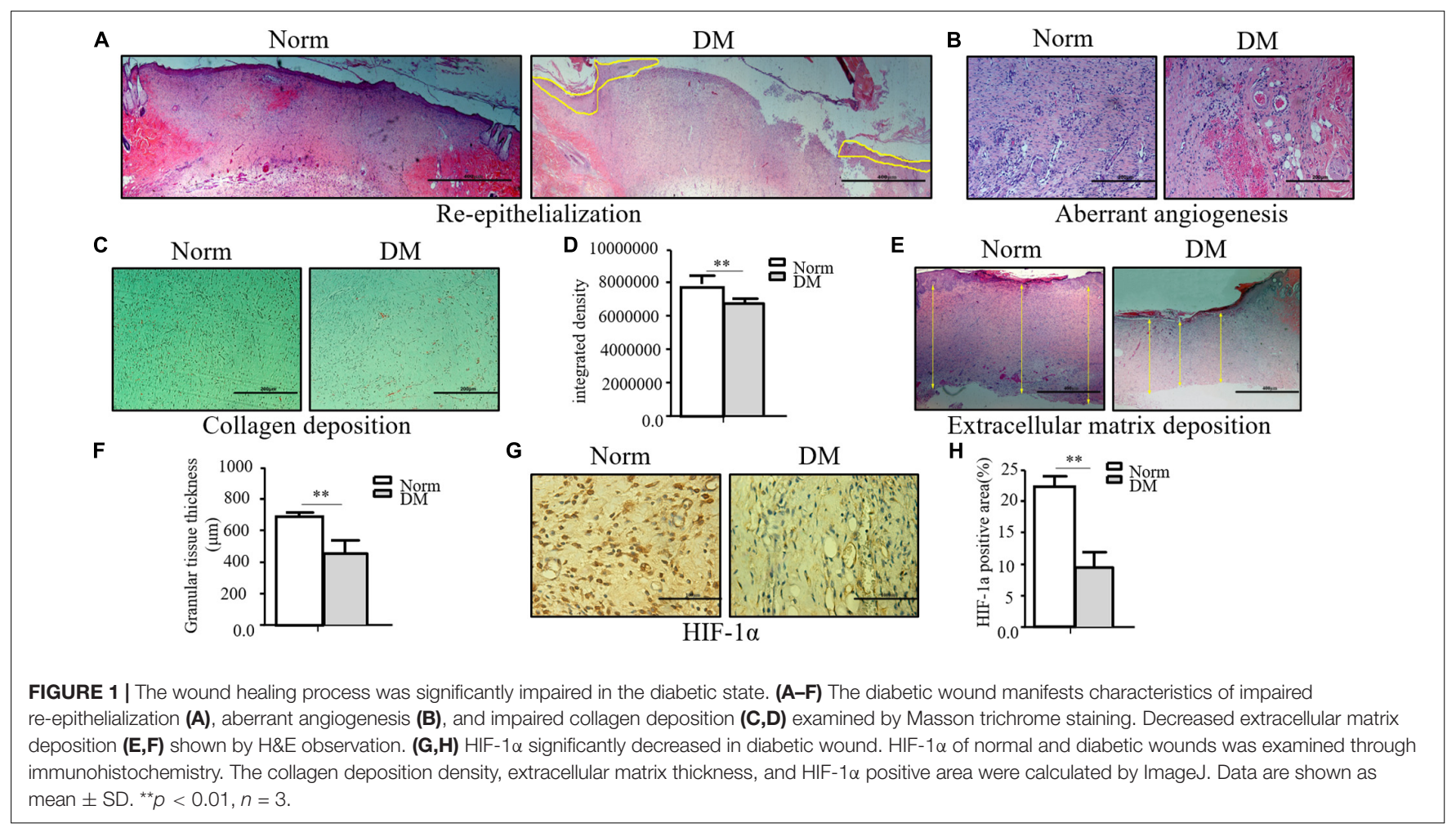



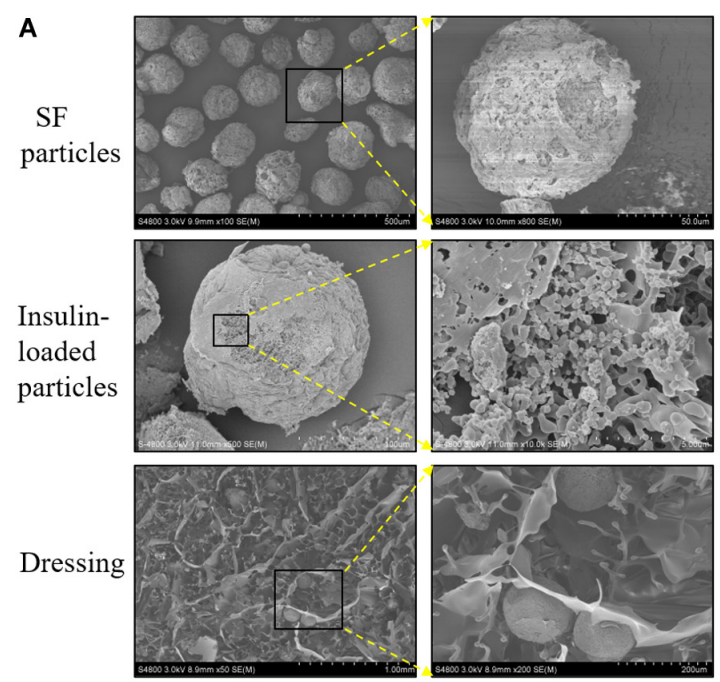

B

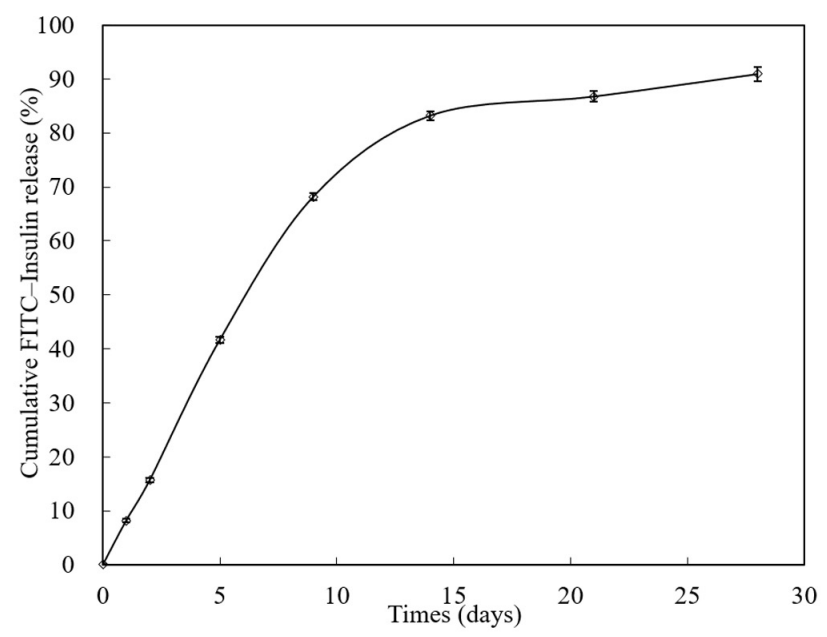

C
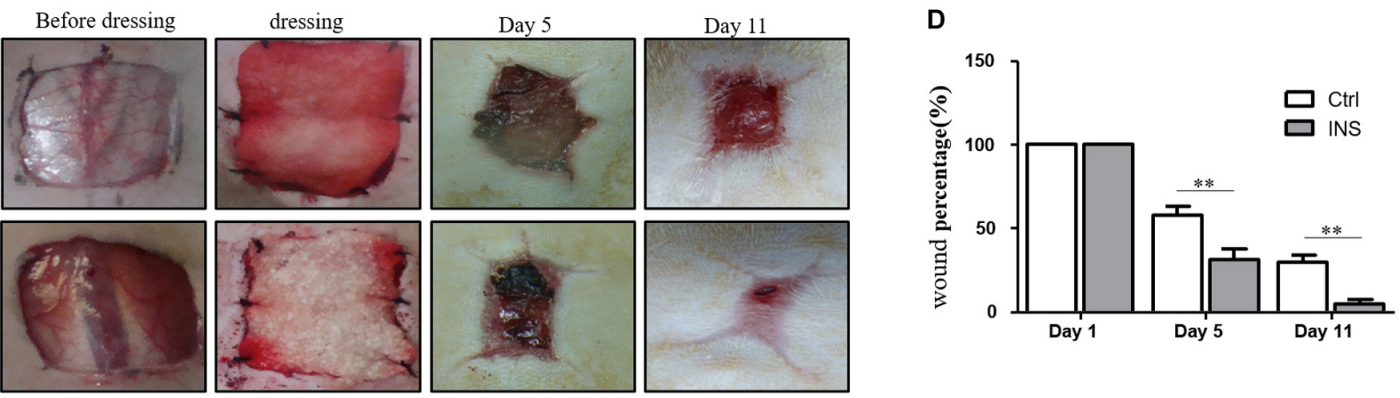

E

Day 5
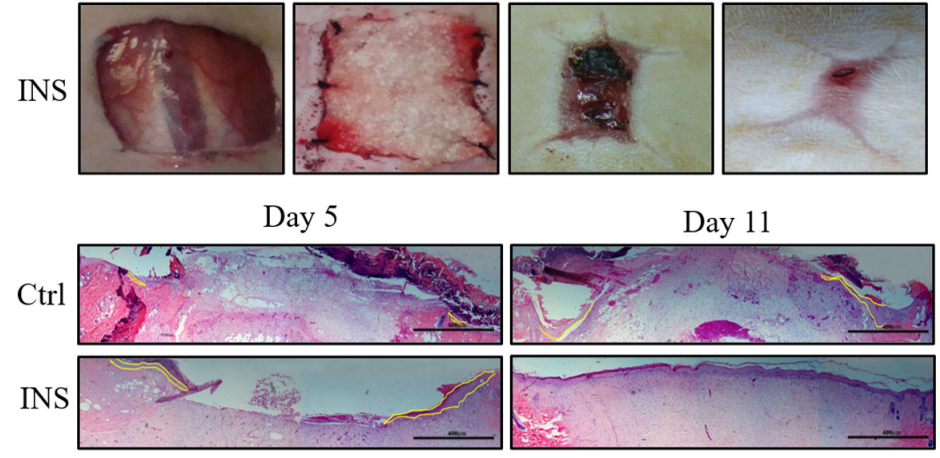

G

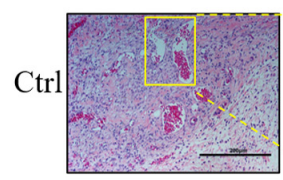

INS

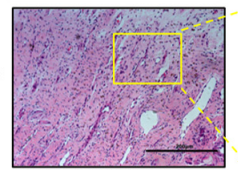

H
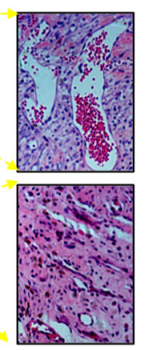
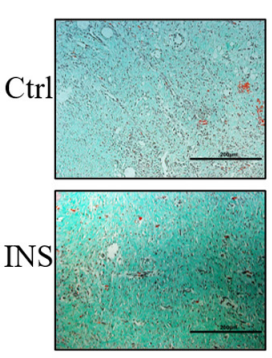
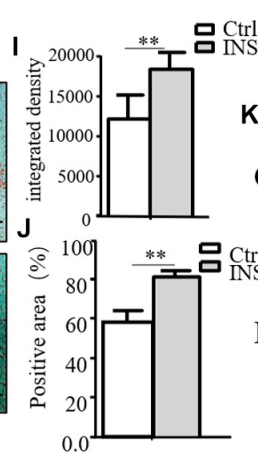

F
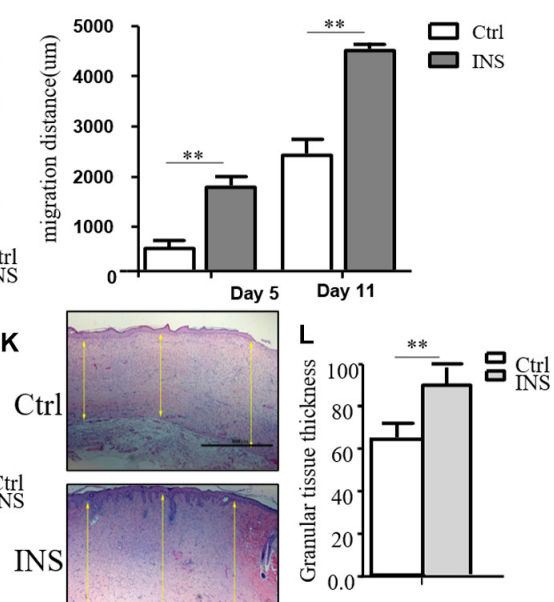

FIGURE 2 | Insulin-containing SF microparticles promote diabetic wound healing. (A) The SEM and cross-sectional views of SF microparticles, insulin-encapsulated SF microparticles, and microparticle-loaded SF. (B) Cumulative FITC-insulin release from microparticle-loaded SF dressings. (C,D) Insulin promoted healing of diabetic wound. After the wounding procedure, the wounds in the control group (Ctrl) were dressed with SF microparticles without insulin; wounds were dressed with the insulin-containing SF microparticles in the insulin group (INS). Wound sizes were recorded on the 1st, 5th, and 11th days after wounding using transparent tracing papers. The unhealed rate of wounds was calculated by Image Pro Plus. Data are shown as mean $\pm \mathrm{SD}$. ${ }^{*} p<0.01, n=5$. (E,F) Insulin-containing dressing enhances reepithelialization of diabetic wounds. Reepithelialization was analyzed on the 5th and 11th day of wounding. The migration length of the tongue was calculated by Image Pro Plus. Data are shown as mean \pm SD. ${ }^{* *} p<0.01, n=5$. (G) Insulin-containing dressing alleviates aberrant angiogenesis of diabetic wounds. Angiogenesis quality of the 5th-day wounds was evaluated by H\&E observation. (H-J) Insulin-containing dressing promotes collagen deposition of diabetic wounds. Masson trichrome staining was used to check collagen deposition in the wound. The integrated density and positive area of collagen deposition were calculated, and data are shown as mean $\pm \mathrm{SD}$. ${ }^{\star *} p<0.01, n=5$. $(\mathbf{K}, \mathbf{L})$ The thickness of the granular tissue was used as a marker of ECM deposition. Data are shown as mean $\pm \mathrm{SD}$. ${ }^{\star *} p<0.01, n=5$. 
material were shown in Figure $\mathbf{2 A}$, and this material could continuously release insulin for more than 20 days (Figure 2B). In this work, it was investigated whether the material promoted diabetic wound healing. As shown in Figures 2C,D, the wound almost healed in the group using insulin-containing dressing (INS) on the 11th day, whereas the control SF microparticles group (Ctrl) still had a large area of non-healing wound. The migration tongues of wounds on the 5th and 11th days also proved that the reepithelialization in the INS group was significantly faster than that in the Ctrl group in Figures 2E,F. Furthermore, the aberrant angiogenesis process in diabetic wounds was also improved in the INS group. Compared with the Ctrl group with the abnormally large and leaky neovascular network, the INS group had normal lumen and healthy vascular network as shown in Figure 2G. The impaired collagen deposition and extracellular matrix deposition were also ameliorated by insulin application as shown in Figures $\mathbf{2 H}-\mathbf{L}$.

\section{MGO Accumulated in the Diabetic State and Played a Toxic Effect on Wound Healing-Related Cells}

Insulin-containing dressing could promote diabetic wound healing, and its mechanism might relate to an improved HIF$1 \alpha$ signal pathway. However, whether insulin affected the HIF- $1 \alpha$ signal pathway to improve the diabetic state is still unknown.
In the diabetic state, many factors influenced the stabilization of HIF- $1 \alpha$, and the accumulation of AGEs was regarded as an indispensable one. MGO was the major form of AGEs and accumulated in the serum and tissue homogenate. As shown in Figure 3A, the MGO level was significantly higher in both serum and dermis in DM than in Norm. We also analyzed the MGO level in high-glucose cultured HUVECs and fibroblasts, which suggested that a higher glucose culture resulted in a higher MGO accumulation in both kinds of cells shown in Figure 3B. Based on the serum MGO level with different concentrations, it is found that MGO inhibited HUVEC and fibroblast proliferation in a concentration-dependent manner. In detail, $800 \mu \mathrm{M}$ of MGO was sufficient to induce the vicious effects on HUVECs and fibroblasts as shown in Figure 3C. Therefore, $800 \mu \mathrm{M}$ of MGO, as optimal concentration, could significantly destabilize HIF- $1 \alpha$ under the hypoxia state in both HUVECs and fibroblasts as shown in Figures 3D-F. Meanwhile, $800 \mu \mathrm{M}$ of MGO also exhibited the effect of inhibiting HUVEC tube formation as shown Figure 3G.

\section{Insulin-Containing Dressing Promotes Diabetic Wound Healing by Stabilizing HIF-1 $\alpha$}

We next investigate whether insulin application could revise these impairments caused by MGO. The results showed that
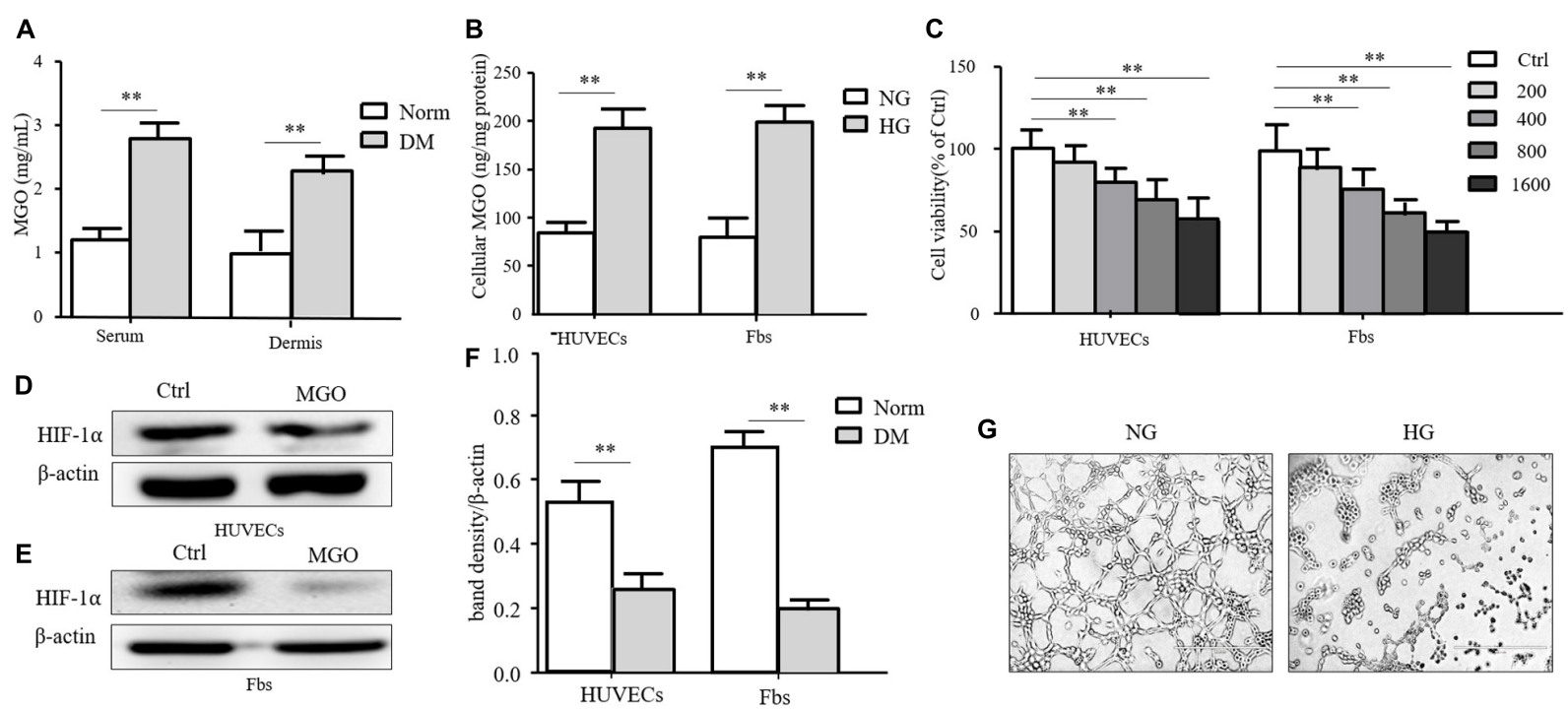

G
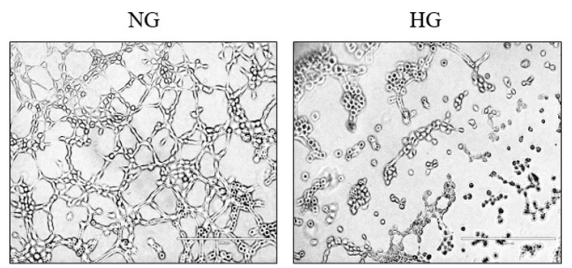

FIGURE 3 | MGO accumulated in the diabetic state and induced an HIF-1 $\alpha$ unstable state. (A) MGO accumulated in the diabetic state. Eight weeks after diabetic induction, the blood and dorsal dermis of diabetic (DM) and normal (Norm) rats were collected. The serum and dermis homogenate were subjected to MGO analysis. Data are shown as mean $\pm \mathrm{SD} .{ }^{* *} p<0.01, n=3$. (B) High glucose incubation leads to MGO accumulation in HUVECs and fibroblasts. Five days after normal glucose (5.5 mM, NG), HUVECs and fibroblasts treated with high glucose (25 mM, HG) were harvested and lysed. The lysis was subjected to MGO analysis. Data are shown as mean $\pm \mathrm{SD}$. ${ }^{* *} p<0.01, n=3$. (C) MGO impaired HUVEC and fibroblast viability in a concentration-dependent manner. HUVECs and fibroblasts were separately treated with 0 (control group), 200, 400, 800, and 1,600 $\mu \mathrm{M} \mathrm{MGO,} \mathrm{and} \mathrm{after} 24 \mathrm{~h}$ of incubation, cells were harvested and subjected to CCK-8 analysis. The viability of each group was compared with that of the control group and presented as the percentage of the control group. Data are shown as mean \pm SD. ${ }^{* *} p<0.01, n=3$. (D-F) MGO induced unstable HIF-1 $\alpha$ in the hypoxia state. After treatment with/without $800 \mu M$ MGO, HUVECs and fibroblasts were subjected to hypoxia ( $1 \%$ oxygen concentration) treatment. Twenty-four hours after treatment, cells were harvested and lysed. The lysis was subjected to western blot analysis for the HIF-1 $\alpha$ level. Photoshop was used in the quantification of immunoblots. Data are shown as mean $\pm \mathrm{SD}$. ${ }^{* *} p<0.01, n=3$. (G) MGO impaired angiogenesis. HUVECs were seeded into a Matrigel with/without $800 \mu \mathrm{M}$ MGO for tube formation analysis. Twelve hours later, the tube formation status in both groups was photographed. 


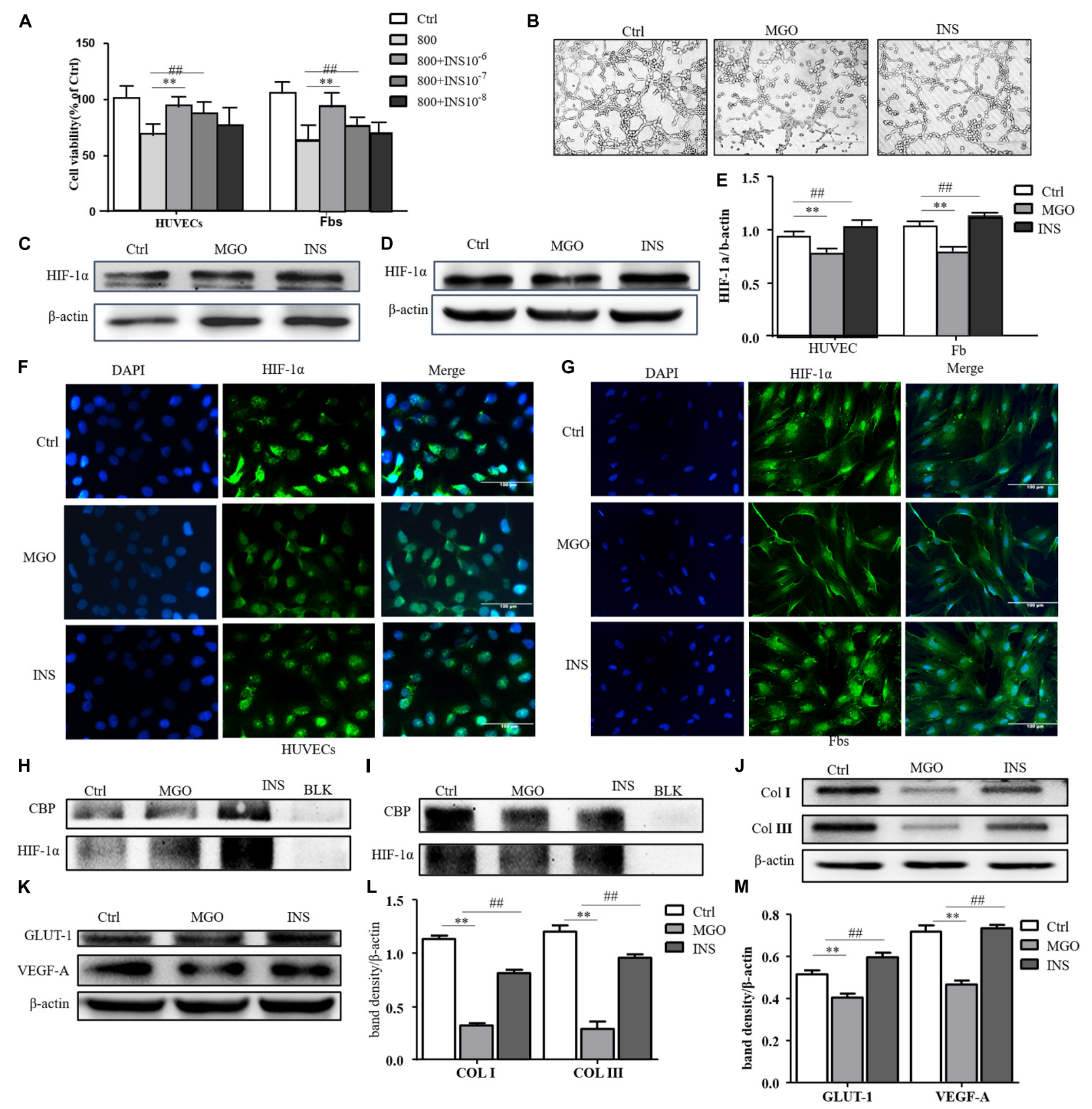

FIGURE 4 | Insulin relieved MGO-induced HIF-1 $\alpha$ impairment in wound healing cells. (A) Insulin promoted MGO-induced cell viability impairment in a concentration-dependent manner. HUVECs and fibroblasts were pretreated with 0 (control group) and $800 \mu \mathrm{M}$ MGO for 30 min. Thirty minutes after MGO treatment, $0,10^{-6}, 10^{-7}$, and $10^{-8} \mathrm{M}$ insulin were added to the culture medium. After $24 \mathrm{~h}$ of incubation, cells were subjected to CCK-8 analysis. The viability of each group was compared with that of the control group and presented as the percentage of the control group. Data are shown as mean $\pm \mathrm{SD}$. ${ }^{* \star} / \# \# p<0.01, n=3$. (B) Insulin promoted MGO-induced HUVEC angiogenesis impairment. HUVECs were set into three groups: control group (Ctrl) treated without MGO and insulin; MGO group (MGO) treated with $800 \mu \mathrm{M}$ MGO; insulin group (INS) treated with $800 \mu \mathrm{M}$ MGO and 10-6 M insulin. The treatment was described previously. Twelve hours later, the tube formation status in both groups was photographed. (C-E) Insulin alleviated MGO-induced HIF-1 $\alpha$ accumulation impairment of HUVECs and fibroblasts. HUVECs and fibroblasts were set as Ctrl, MGO, and INS groups and treated as described above. Twenty-four hours under the hypoxia state (1\% oxygen concentration), cells were harvested, and HIF-1 $\alpha$ levels were analyzed. Photoshop was used in the quantification of immunoblots. Data are shown as mean \pm SD. **/\#\#p < 0.01, $n=3$. *Means Ctrl vs. MGO; \#means MGO vs. INS. (F,G). Insulin promoted HIF-1 $\alpha$ translocation into HUVECs and fibroblasts. In Ctrl, MGO, and INS groups and after $24 \mathrm{~h}$ under the hypoxia state, cells were harvested and fixed with $4 \%$ paraformaldehyde. The cells were then subjected to immunofluorescence examination to analyze the HIF-1 $\alpha$ accumulation and translocation to the nucleus. (H,I) Insulin promoted translation complex formation of HIF-1 $\alpha$ and CBP in HUVECs and fibroblasts. Cell lysis of Ctrl, MGO, and INS was subjected to co-IP examination to analyze the HIF-1 $\alpha$ and CBP complex formation. (J-M) Insulin improved MGO-induced HIF-1 $\alpha$ pathway impairment in HUVECs and fibroblasts. Cell lysis of Ctrl, MGO, and INS was subjected to western blot to examine the expression of HIF-1 $\alpha$ target genes VEGF-A and GLUT-1 (HUVECs) and collagen I and collagen III (fibroblasts). Photoshop was used in the quantification of immunoblots. Data are shown as mean $\pm \mathrm{SD}$. ${ }^{\star *} / \# \# p<0.01, n=5$. *Means Ctrl vs. MGO; \#means MGO vs. INS. 
insulin could revise inhibitions of HUVEC and fibroblast proliferation in a concentration-dependent manner as shown in Figure 4A. Meanwhile, $10^{-6} \mathrm{mM}$ of insulin, as optimal concentration, could significantly alleviate MGOinduced HUVEC tube formation impairment as shown in Figure 4B. Furthermore, the instability of HIF-1 $\alpha$ was also significantly revised in the insulin application group through immunofluorescence. In detail, co-IP of HIF- $1 \alpha$ and CBP, the signature of HIF- $1 \alpha$, exerted their transcription functions (Figures 4C-I), and protein expression of HIF-1 $\alpha$ target genes was significantly enhanced as shown in Figures 4J-M. The results showed that insulin promoted HIF-1 $\alpha$ stabilization by the translocating into the nucleus.

\section{Insulin-Containing Material Promotes Wound Healing Through HIF-1 $\alpha$ and Its Downstream Effector}

We confirmed in this work that insulin application could reverse MGO-induced HIF- $1 \alpha$ instability and promote its signal effect,

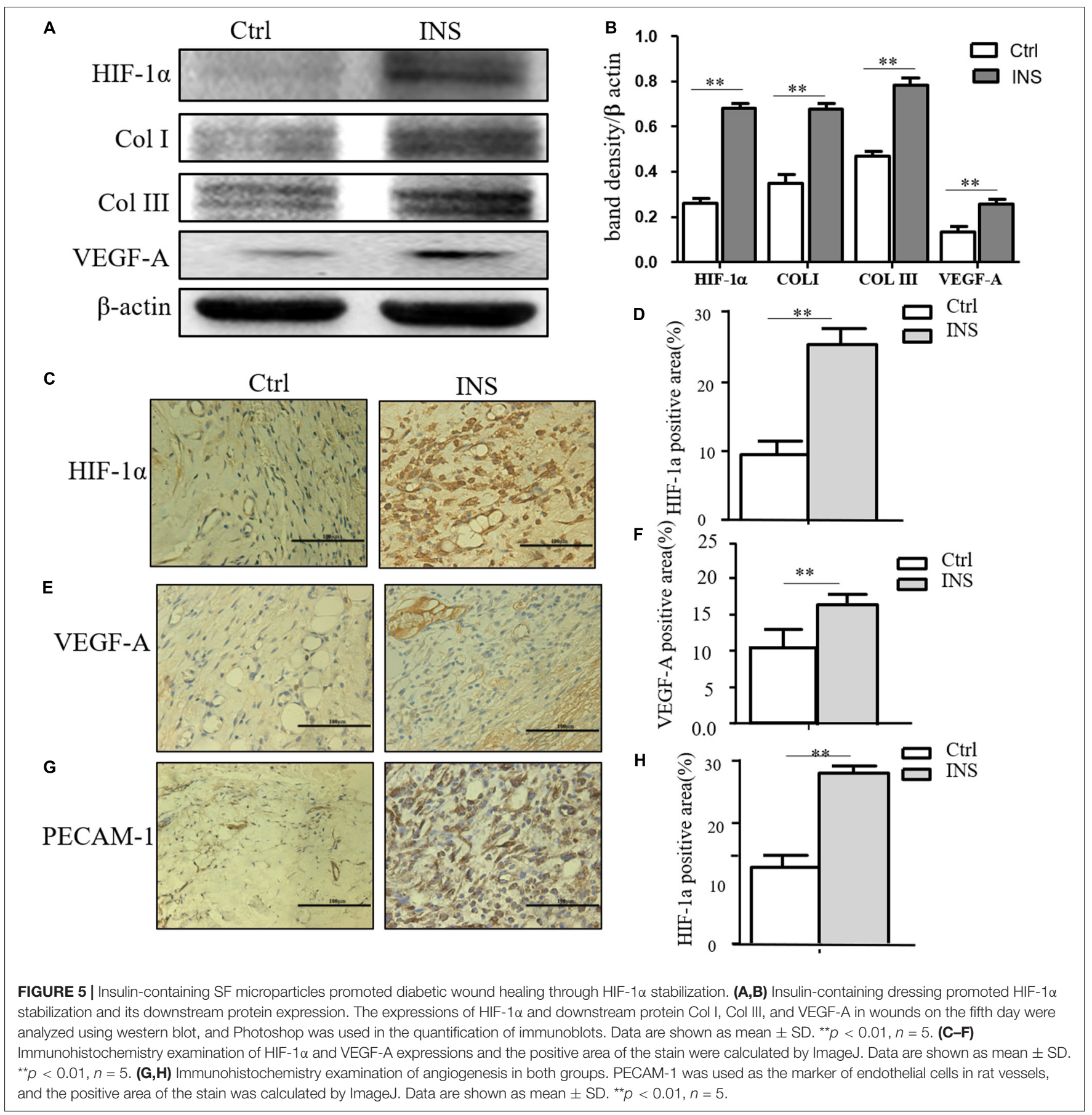


an effect of insulin-containing material treating wounds. HIF$1 \alpha$ and its downstream effector including VEGF-A, GLUT-1, Col I, and Col III were significantly higher in insulincontaining dressing wounds as shown in Figures $\mathbf{5 A , B}$. The immunochemistry examination of VEGF-A, vessel density, and collagen deposition further proved this assumption (Figures 5C,D).

\section{DISCUSSION}

Once a wound formed, the healing process initiates. Due to the destruction of blood supply and the high proliferation potential of wound cells, the wound is in an absolute or relative hypoxia state during a considerable part of the time. The process of wound healing in other words is the recovery of tissue structure and oxygen supply. HIF- $1 \alpha$ plays a very important role in this process and is constitutively expressed in most cells. However, it is very sensitive to oxygen and would be degraded by the ubiquitin-proteasome immediately under a normoxia state. Under the hypoxia state in the wound healing environment, HIF$1 \alpha$-degrading pathways are inhibited, and HIF- $1 \alpha$ accumulates in the cytoplasm due to the inhibited HIF-1 $\alpha$-degrading pathways, and then it translocates into the nucleus to exert its translation factor function. When forming a transcription complex with HIF-1 $\beta$, the co-activator CBP initiates a number of gene expression targets to inflammation reaction, angiogenesis, reepithelialization, and extracellular matrix deposition (Zhao et al., 2012). Under the diabetic state, high glucose and its side-products can inhibit the stability of HIF-1 $\alpha$ in the hypoxia environment in many ways. MGO is a by-product of cell glucose metabolism in the condition of diabetes mellitus and has a significantly increased concentration in both rats and highglucose cultured cells. Results showed that MGO can inhibit HIF- $1 \alpha$ signal pathways in many ways such as downregulating the stability of HIF- $1 \alpha$ through enhancing the chaperone-dependent ubiquitin degradation of HIF-1 $\alpha$ (Bento et al., 2010), promoting the activity of PHD activation, and influencing the combination of HIF- $1 \alpha$ and HIF- $1 \beta$ or HIF- $1 \alpha$ and CBP (Bento and Pereira, 2011). Our results show that MGO affects the stability of HIF- $1 \alpha$ and decreases the binding of HIF- $1 \alpha$ and CBP. The significantly decreased protein levels of HIF- $1 \alpha$ and its downstream target genes after MGO treatment further proved the vicious role that MGO plays in the HIF-1 $\alpha$-mediated wound healing process. The impairment of the HIF-1 $\alpha$ pathway was considered as a pivotal mechanism of diabetic non-healing wounds. Promoting the

\section{REFERENCES}

Bento, C. F., Fernandes, R., Ramalho, J., Marques, C., Shang, F., Taylor, A., et al. (2010). The chaperone-dependent ubiquitin ligase CHIP targets HIF-1alpha for degradation in the presence of methylglyoxal. PLoS One 5:e15062. doi: 10.1371/journal.pone.0015062

Bento, C. F., and Pereira, P. (2011). Regulation of hypoxia-inducible factor 1 and the loss of the cellular response to hypoxia in diabetes. Diabetologia 54, 1946-1956. doi: 10.1007/s00125-011-2191-8 stability of HIF- $1 \alpha$ is an important research direction targeting at promotion of diabetic wound healing (Catrina and Zheng, 2016). Our previous researches confirm that insulin can promote wound healing by promoting epidermal migration, angiogenesis, extracellular matrix deposition, etc., which are exactly the downstream effects of HIF- $1 \alpha$. These results focus on HIF- $1 \alpha$ and its downstream pathway. The application of insulin can promote the accumulation of HIF- $1 \alpha$ in the cytoplasm and nucleus, and then HIF-1 $\alpha$ will combine with CBP to activate its downstream transcription. In summary, this study confirmed that the increase of MGO content in diabetes can lead to the instability of HIF- $1 \alpha$, which results in the delay of wound healing due to the damage of the HIF- $1 \alpha$ signal pathway and its downstream effects. Wound dressing containing insulin can stabilize HIF-1 $\alpha$, promote the downstream effects related to HIF-1 $\alpha$, and enhance the healing of diabetic wound.

\section{DATA AVAILABILITY STATEMENT}

The raw data supporting the conclusions of this article will be made available by the authors, without undue reservation.

\section{ETHICS STATEMENT}

The animal study was reviewed and approved by Animal Care Committee of SJTUSM.

\section{AUTHOR CONTRIBUTIONS}

All authors listed have made a substantial, direct and intellectual contribution to the work, and approved it for publication.

\section{FUNDING}

This study was supported by the project National Natural Science Foundation of China (81170761, 81270909, and 81871564), Shanghai Hospital Development Center (SHDC12014117), Shanghai Municipal Key Clinical Specialty (shslczdzk02302), Shanghai Chinese and Western Medicine Clinical Cooperation Pilot Cultivation Project [ZY(2018-2020)-FWTX-1106], Ruijin Youth NSFC Cultivation Fund (2019QNPY01003), and Shanghai Jiao Tong University Medical Engineering Cross Research Funds (YG2019QNA35).

Botusan, I. R., Sunkari, V. G., Savu, O., Catrina, A. I., Grunler, J., Lindberg, S., et al. (2008). Stabilization of HIF-1alpha is critical to improve wound healing in diabetic mice. Proc. Natl. Acad. Sci. U.S.A. 105, 19426-19431. doi: 10.1073/ pnas.0805230105

Catrina, S. B. (2014). Impaired hypoxia-inducible factor (HIF) regulation by hyperglycemia. J. Mol. Med. 92, 1025-1034. doi: 10.1007/s00109-014-1166-x

Catrina, S. B., and Zheng, X. (2016). Disturbed hypoxic responses as a pathogenic mechanism of diabetic foot ulcers. Diabetes Metab. Res. Rev. 32(Suppl. 1), 179-185. doi: 10.1002/dmrr.2742 
Davis, F. M., Kimball, A., Boniakowski, A., and Gallagher, K. (2018). Dysfunctional wound healing in diabetic foot ulcers: new crossroads. Curr. Diab. Rep. 8:2.

Demidova-Rice, T. N., Hamblin, M. R., and Herman, I. M. (2012). Acute and impaired wound healing: pathophysiology and current methods for drug delivery, part 1: normal and chronic wounds: biology, causes, and approaches to care. Adv. Skin Wound Care 25, 304-314. doi: 10.1097/01.asw.0000416006. 55218.d0

Falanga, V. (2005). Wound healing and its impairment in the diabetic foot. Lancet 366, 1736-1743. doi: 10.1016/s0140-6736(05)67700-8

Hong, W. X., Hu, M. S., Esquivel, M., Liang, G. Y., Rennert, R. C., McArdle, A., et al. (2014). The role of hypoxia-inducible factor in wound healing. Adv. Wound Care 3, 390-399. doi: 10.1089/wound.2013.0520

Junod, A., Lambert, A. E., Stauffacher, W., and Renold, A. E. (1969). Diabetogenic action of streptozotocin: relationship of dose to metabolic response. J. Clin. Invest. 48, 2129-2139. doi: 10.1172/jci106180

Li, X., Liu, Y., Zhang, J., You, R., Qu, J., and Li, M. (2017). Functionalized silk fibroin dressing with topical bioactive insulin release for accelerated chronic wound healing. Mater Sci. Eng. C Mater Biol. Appl. 72, 394-404. doi: 10.1016/j. msec.2016.11.085

Liu, Y., Petreaca, M., and Martins-Green, M. (2009a). Cell and molecular mechanisms of insulin-induced angiogenesis. J Cell Mol Med. 13, 4492-4504. doi: $10.1111 / j .1582-4934.2008 .00555 . x$

Liu, Y., Petreaca, M., Yao, M., and Martins-Green, M. (2009b). Cell and molecular mechanisms of keratinocyte function stimulated by insulin during wound healing. BMC Cell Biol. 10:1. doi: 10.1186/1471-2121-10-1
Mace, K. A., Yu, D. H., Paydar, K. Z., Boudreau, N., and Young, D. M. (2007). Sustained expression of Hif-1alpha in the diabetic environment promotes angiogenesis and cutaneous wound repair. Wound Repair Regen. 15, 636-645. doi: 10.1111/j.1524-475x.2007.00 278.x

Ramasamy, R., Yan, S. F., and Schmidt, A. M. (2006). Methylglyoxal comes of AGE. Cell 124, 258-260. doi: 10.1016/j.cell.2006. 01.002

Zhao, Y. Z., Li, X., Lu, C. T., Xu, Y. Y., Lv, H. F., Dai, D. D., et al. (2012). Experiment on the feasibility of using modified gelatin nanoparticles as insulin pulmonary administration system for diabetes therapy. Acta Diabetol. 49, 315-325. doi: 10.1007/s00592-011-0 $356-\mathrm{z}$

Conflict of Interest: The authors declare that the research was conducted in the absence of any commercial or financial relationships that could be construed as a potential conflict of interest.

Copyright () 2020 Yang, Wang, Shi, Li, Gao, Yu, Liu, Zhang, Wang, Zhang and Liu. This is an open-access article distributed under the terms of the Creative Commons Attribution License (CC BY). The use, distribution or reproduction in other forums is permitted, provided the original author(s) and the copyright owner(s) are credited and that the original publication in this journal is cited, in accordance with accepted academic practice. No use, distribution or reproduction is permitted which does not comply with these terms. 